\title{
Correction to: Associations between serumlL-8 and knee symptoms, joint structures, and cartilage or bone biomarkers in patients with knee osteoarthritis
}

\author{
Guangfeng Ruan ${ }^{1,2,3} \cdot$ Changhai Ding ${ }^{1,2,3}$ \\ Published online: 28 May 2020 \\ (C) International League of Associations for Rheumatology (ILAR) 2020
}

\section{Correction to: Clinical Rheumatology (2019) 38:3609-3617 https://doi.org/10.1007/s10067-019-04718-8}

It has been brought to our attention that there were overlaps between the article recently published in this journal [1] and the previous publications from our group [2-4] that we did not cite. We apologize to the readers for omitting the citations; however, the overlaps were reasonable and not all avoidable.

First, the overlaps between these articles were mainly in the methods section, which caused by the fact that these articles were using the same sample, the same design, and the similar outcomes. Moreover, the exposures were all serum cytokines measured by the same methods. Therefore, these overlaps were not all avoidable.

Second, there were a few of overlaps in the result section due to the fixed descriptive sentences (e.g., "were significantly and positively associated with total WOMAC score before and after adjustment for age, sex and BMI"), but the overlaps were not substantial.

The online version of the original article can be found at https://doi.org/ 10.1007/s10067-019-04718-8

Changhai Ding

changhai.ding@utas.edu.au

Guangfeng Ruan

ruan1989.ok@163.com

1 Department of Rheumatology and Immunology, Arthritis Research Institute, the First Affiliated Hospital of Anhui Medical University, No. 218, Jixi Street, Hefei, Anhui, China

2 Menzies Institute for Medical Research, University of Tasmania, 17 Liverpool Street, Hobart, Tasmania, Australia

3 Clinical Research Centre, Zhujiang Hospital, Southern Medical University, No. 253, Middle Gongye Avenue,

Guangzhou, Guangdong, China
Third, there were minor overlaps in the introduction and/or discussion sections. All these articles were about knee osteoarthritis, with similar research background and rationale. Particularly, these articles were focusing on inflammatory factors, which means there would be some shared causal pathways.

Overall, this article reported that serum IL-8 was associated with knee symptoms, joint structures, and cartilage or bone biomarkers in patients with knee osteoarthritis, which were the first study to describe these associations. Some overlaps mainly in the methods section with our previous publications would not affect the novelty and integrity of this publication.

\section{References}

1. Ruan G, Xu J, Wang K, Zheng S, Wu J, Bian F, Chang B, Zhang Y, Meng T, Zhu Z, Han W, Ding C (2019) Associations between serum IL-8 and knee symptoms, joint structures, and cartilage or bone biomarkers in patients with knee osteoarthritis. Clin Rheumatol 38(12): 3609-3617

2. Ruan G, Xu J, Wang K, Zheng S, Wu J, Ren J, Bian F, Chang B, Zhu Z, Han W, Ding C (2019) Associations between serum S100A8/ S100A9 and knee symptoms, joint structures and cartilage enzymes in patients with knee osteoarthritis. Osteoarthr Cartil 27(1):99-105

3. Wu J, Wang K, Xu J, Ruan G, Zhu Q, Cai J, Ren J, Zheng S, Zhu Z, Otahal P, Ding C (2017) Associations between serum ghrelin and knee symptoms, joint structures and cartilage or bone biomarkers in patients with knee osteoarthritis. Osteoarthr Cartil 25(9):1428-1435

4. Ruan G, Xu J, Wang K, Wu J, Zhu Q, Ren J, Bian F, Chang B, Bai X, Han W, Ding C (2018) Associations between knee structural measures, circulating inflammatory factors and MMP13 in patients with knee osteoarthritis. Osteoarthr Cartil 26(8):1063-1069

Publisher's note Springer Nature remains neutral with regard to jurisdictional claims in published maps and institutional affiliations. 